OPEN ACCESS

Edited by:

Yuji Morita,

Aichi Gakuin University, Japan

Reviewed by:

Juan M. Tomas,

University of Barcelona, Spain

Holly A. Sinclair,

Pathology Queensland, Australia

*Correspondence:

Wen-Chien Ko

winston3415@gmail.com

Specialty section:

This article was submitted to

Infectious Diseases,

a section of the journal

Frontiers in Microbiology

Received: 23 March 2016 Accepted: 11 May 2016

Published: 27 May 2016

Citation:

Chen P-L, Lamy B and Ko W-C (2016) Aeromonas dhakensis, an Increasingly Recognized Human

Pathogen. Front. Microbiol. 7:793. doi: 10.3389/fmicb.2016.00793

\section{Aeromonas dhakensis, an Increasingly Recognized Human Pathogen}

\author{
Po-Lin Chen ${ }^{1,2}$, Brigitte Lamy ${ }^{3,4}$ and Wen-Chien Ko $\mathrm{K}^{1,2 *}$ \\ 1 Department of Internal Medicine, National Cheng Kung University Hospital, College of Medicine, National Cheng Kung \\ University, Tainan, Taiwan, ${ }^{2}$ Center for Infection Control, National Cheng Kung University Hospital, Tainan, Taiwan, \\ ${ }^{3}$ Laboratoire de Bactériologie-Virologie, Équipe Pathogènes Hydriques Santé Environnements, UMR 5569 HydroSciences \\ Montpellier, Université de Montpellier, Montpellier, France, ${ }^{4}$ Laboratoire de Bactériologie, Centre Hospitalier Régional \\ Universitaire de Montpellier, Montpellier, France
}

Aeromonas dhakensis was first isolated from children with diarrhea in Dhaka, Bangladesh and described in 2002. In the past decade, increasing evidence indicate this species is widely distributed in the environment and can cause a variety of infections both in human and animals, especially in coastal areas. A. dhakensis is often misidentified as $A$. hydrophila, $A$. veronii, or $A$. caviae by commercial phenotypic tests in the clinical laboratory. Correct identification relies on molecular methods. Increasingly used matrix-assisted laser desorption ionization-time of flight mass spectrometry (MALDI-TOF MS) may be able to identify Aeromonas specie rapidly and accurately. A. dhakensis has shown its potent virulence in different animal models and clinical infections. Although several virulence factors had been reported, no single mechanism is conclusive. Characteristically $A$. dhakensis is the principal species causing soft tissue infection and bacteremia, especially among patients with liver cirrhosis or malignancy. Of note, $A$. dhakensis bacteremia is more lethal than bacteremia due to other Aeromonas species. The role of this species in gastroenteritis remains controversial. Third generation cephalosporins and carbapenems should be used cautiously in the treatment of severe A. dhakensis infection due to the presence of AmpC $\beta \beta$-lactamase and metallo$\beta$-lactamase genes, and optimal regimens may be cefepime or fluoroquinolones. Studies of bacterial virulence factors and associated host responses may provide the chance to understand the heterogeneous virulence between species. The hypothesis $A$. dhakensis with varied geographic prevalence and enhanced virulence that compared to other Aeromonas species warrants more investigations.

\footnotetext{
Keywords: Aeromonas dhakensis, taxonomy, identification, epidemiology, clinical infection, virulence, antimicrobial resistance
}

The genus Aeromonas includes ubiquitous bacteria found in aquatic habitats. Among the numerous Aeromonas species that have been described, at least 31 species until March 2016, Aeromonas dhakensis has a particular position. It has been mistaken for decades as A. hydrophila, been a part of the taxonomical controversies in the genus Aeromonas, and exhibits characteristics for marked virulence. Indeed, no other species description has led to such a revision in our knowledge on epidemiology and virulence understanding of the genus Aeromonas. Here, we 
performed a systematic review on $A$. dhakensis, including taxonomical, epidemiological, clinical, pathogenic and therapeutic issues, and focus on clinical infectious diseases, as the latest research showed growing evidence of the major role of $A$. dhakensis in human infections.

\section{HISTORY OF TAXONOMY FOR Aeromonas dhakensis}

Aeromonas hydrophila subsp. dhakensis, isolated from children with diarrhea in Dhaka, Bangladesh between 1993 and 1994, was first reported by Huys et al. (2002). These A. hydrophila-like strains initially referred to as the group BD-2 were most closely related to A. hydrophila DNA hybridization group (HG) 1, but examination of 152 phenotypic characteristics revealed that the group BD-2 isolates differed from the representatives of HG1 in eight biochemical properties. Martinez-Murcia et al. (2008) analyzed the strains isolated from water and skin of ornamental fish from Portugal by polyphasic approaches, including $g y r B$, $r p o D$, and 16S rRNA gene sequencing, DNA-DNA hybridization, matrix-assisted laser desorption ionization-time of flight mass spectrometry (MALDI-TOF MS) analysis, genotyping by random amplified polymorphic DNA, and extensive biochemical and antibiotic susceptibility tests. Martinez-Murcia et al. (2008) proposed a new species, A. aquariorum sp. nov.. However, 1 year later, phylogenetic analysis of three strains of $A$. hydrophila subsp. dhakensis using $r p o D$ and gyrB sequencing showed that they shared the same taxon with $A$. aquariorum (MartinezMurcia et al., 2009). Further phylogenetic study derived from $16 \mathrm{~S}$ rRNA, rpoD or gyrB genes and a multilocus phylogenetic analysis (MLPA; with the concatenated sequences of $g y r B, r p o D$, $\operatorname{rec} A$, dnaJ, and gyrA) by Beaz-Hidalgo et al. (2013) confirmed that $A$. aquariorum and A. hydrophila subsp. dhakensis are the same taxon, and are different from the A. hydrophila taxon. Accordingly, formal A. aquariorum and A. hydrophila subsp. dhakensis have been reclassified as $A$. dhakensis sp. nov. comb nov. (Beaz-Hidalgo et al., 2013). Since then, whole genome sequence analyses unambiguously confirmed that $A$. dhakensis reached the level of species and showed that many strains have been misidentified as A. hydrophila (Colston et al., 2014; BeazHidalgo et al., 2015).

\section{CHARACTERISTICS OF A. dhakensis}

Aeromonas dhakensis strains, like other Aeromonas species, have typical Aeromonas characteristics: motile gram-negative bacilli, chemoorganotrophs with both oxidative and fermentative metabolism, cytochrome oxidase- and catalase-positivity, reduction of nitrate to nitrite without gas production, and resistance to the vibriostatic agent O/129 (Huys et al., 2002). They optimally grow after $24 \mathrm{~h}$ at $28^{\circ} \mathrm{C}$ and can also grow at $42^{\circ} \mathrm{C}$ on TSA medium. The phenotypic characterization of A. dhakensis differs from two other A. hydrophila subspecies (A. hydrophila subsp. hydrophila or ranae) by at least three biochemical properties, namely utilization of urocanic acid and
L-arabinose, and acid production from L-arabinose (Huys et al., 2002, 2003).

\section{SPECIES IDENTIFICATION}

Aeromonas dhakensis is often clinically misidentified as A. hydrophila by phenotypic methods (Figueras et al., 2009). $16 \mathrm{~S}$ rRNA sequencing has been used for more than two decades in identifying Aeromonas to the genus level (Martinez-Murcia et al., 1992), but the bulk 16S rRNA sequences are unreliable in identifying Aeromonas to the species level (Janda and Abbott, 2007). Indeed, there is on one hand a very low variability of the 16S rRNA sequence for closely related species and, on the other hand, some heterogeneity in sequences between rrn operons, both at an intra-genomic level (variability between copies within a given genome), an intra-species level (between strains within a given species) and an inter-species level. This hampers identification based on bulk $16 \mathrm{~S}$ rRNA sequences (Roger et al., 2012a). Even when taking into account the multioperon diversity of $16 \mathrm{~S}$ rRNA, no specific signature could be identified for $A$. dhakensis and combinations of sequences could not unambiguously distinguish $A$. dhakensis from $A$. caviae (Roger et al., 2012a). Correct identification can be achieved using nucleotide sequencing of housekeeping genes, such as rpoB, rpoD, or gyrB (Martinez-Murcia et al., 2009; Wu et al., 2012b). Several studies showed that MLPA based on several housekeeping genes improves discriminative power (Martinez-Murcia et al., 2011; Martino et al., 2011; Roger et al., 2012b). For example, Martinez-Murcia et al. (2011) showed that it was achievable to clarify the genetic divergence corresponding to the intra-species or inter-species levels, based on gyrB, rpoD, recA, dnaJ, gyrA, dnaX, and atpD.

Several studies have shown that MALDI-TOF MS could efficiently identify Aeromonas species (Donohue et al., 2007; Lamy et al., 2011). Taxonomic identification of $A$. dhakensis by MALDI-TOF MS was firstly reported by Martinez-Murcia et al. (2008), and our work analyzing 30 clinical A. dhakensis isolates found that accuracy rate of MALDI-TOF MS was 96.7\% (Chen et al., 2014a). However, A. dhakensis is not yet included in the commercial database of the MALDI-TOF system and may be reported as A. hydrophila, A. veronii, A. caviae, or A. bestiarum (Chen et al., 2014a).

\section{GLOBAL DISTRIBUTION OF A. dhakensis}

Aeromonas dhakensis has been isolated from clinical specimens, animals, and environment in different countries with varying frequency and water is likely its primary habitat. A. dhakensis is widely distributed in tropical and subtropical areas. In environments, this species has been recovered from river water, cooling-system water pond, and fish tank water (MartinezMurcia et al., 2008; Esteve et al., 2012). In food, A. dhakensis has been recovered from marine shrimps in low salinity ponds in Thailand (18\% of 70 Aeromonas isolates, Yano et al., 2015). In addition, $A$. dhakensis was isolated from diseased eels (Esteve 
et al., 2012), septicemic ornemental fish (Jagoda et al., 2014), a diseased dolphin in Spain (Perez et al., 2015), chironomid egg masses in Israel (Figueras et al., 2011), and diseased fish in south Korea (Yi et al., 2013) and Mexico (Soto-Rodriguez et al., 2011). Of note, it was the predominant species, accounting for $31.4 \%$ of the Aeromonas isolates cultivated from farm eels in Korea (Yi et al., 2013).

\section{CLINICAL EPIDEMIOLOGY}

With the warming climate, this species may become more prevalent in the environment along with an increased risk of exposure. Although the global epidemiology study of $A$. dhakensis is lacking, current studies show that it is prevalent in countries with hot climate, such as Bangladesh (Huys et al., 2002), Taiwan (Chen et al., 2014c; Wu et al., 2015), Australia (Aravena-Roman et al., 2011), and Malaysia (Puthucheary et al., 2012). The virulent A. hydrophila SSU strain recently reclassified as A. dhakensis SSU was originally isolated from a patient with diarrhea in Philippines (Grim et al., 2014). A case of acute gastroenteritis after returning from Egypt attributed to A. dhakensis occurred in Czech Republic and was concluded as an imported disease (Sedlacek et al., 2012). In countries with temperate climate, clinical infection caused by this species is rarely reported. For example, only one fatal A. dhakensis sepsis was reported in a cirrhotic Korean (Shin et al., 2013). Four cases of $A$. dhakensis bloodstream infection were reported in Nagasaki, Japan (Morinaga et al., 2011a). In the Roger's study enrolling 89 and 13 clinical isolates from mainland France and its overseas territories, respectively, 5.9\% (six isolates) belonged to the species $A$. dhakensis. Interestingly, four of them were recovered from a tropical or subtropical origin, e.g., either French West Indies or Reunion Island or from travelers returning from these areas (Roger et al., 2012b). However, the present information about the global distribution of $A$. dhakensis in environment and human is very likely to be underrepresented. With the increasing availability of sophisticated diagnostic tools, accurate taxonomy can improve the visibility of $A$. dhakensis in nature and patients.

\section{VIRULENCE MECHANISMS of A. dhakensis}

The pathogenicity of Aeromonas species has been considered multifactorial. A number of virulence factors, such as secretion system, toxins, and quorum sensing system (QSS), have been discovered in the past 30 years (Tomas, 2012). The pathogenicity studies specific for $A$. dhakensis are increasing, owing to its clinical virulence and recent reclassification of taxonomy. There is converging evidence suggesting that A. dhakensis exhibits greater virulence compared to other Aeromonas species.

Aeromonas dhakensis (previously named A. aquariorum) strains have been noted to possess cytotoxic activities against human blood cell lines (Morinaga et al., 2011b) and three isolates recovered from diseased eels were virulent to healthy eels (Esteve et al., 2012). In our previous work comparing four major
Aeromonas species associated clinical infections, A. dhakensis was more virulent than the other three species, i.e., A. veronii, A. caviae, and A. hydrophila, in terms of mouse fibroblast $\mathrm{C} 2 \mathrm{C} 12$ cell line, BALB/c mouse, and Caenorhabditis elegans models (Chen et al., 2014d). Despite limited number of strains, Mosser et al. (2015) found that $A$. dhakensis exhibited high virulence in the $C$. elegans infection model, with unusual presentation of rapid lysis of dead body by $A$. dhakensis BVH28b. Similar events caused by the strain $A$. dhakensis AAK1 was observed in our previous work (Chen et al., 2014d). The mechanisms used by A. dhakensis to rapidly kill worms remain unclear (Mosser et al., 2015) and deserve to be further studied. With the progress of next generation sequencing, comparative genomics coupled with functional assays allow us to investigate the pathogen specific markers or virulence factors in the clinical or environment strains.

Over the past decades, several virulence factors and associated secretion systems have been comprehensively studied in a clinical strain, A. dhakensis SSU (formerly classified as A. hydrophila SSU). This makes $A$. dhakensis one of the most studied species for its virulence within the genus Aeromonas together with A. salmonicida. The proposed virulence factors of $A$. dhakensis SSU are summarized in Table 1. For example, heat-labile cytotoxic enterotoxin (Alt) and heat-stable cytotoxic enterotoxin (Ast) have been linked to clinical gastroenteritis, as both could increase cAMP levels in intestinal mucosa (Chopra and Houston, 1999). An aerolysin-related cytotoxic enterotoxin (Act) secreted by the type 2 secretion system (T2SS) is able to cause diarrheal diseases and deep wound infections in mice (Xu et al., 1998; Sha et al., 2002). A type 3 secretion system effector, AexU, with ADP-ribosyltransferase and GAP activities, plays a role in the pathogenesis of Aeromonas infection (Sha et al., 2007). Moreover, the type 6 secretion system (T6SS) has proved crucial in virulence through many studies in A. dhakensis SSU (Suarez et al., 2008, 2010b). A T6SS secreted effector, hemolysin coregulated protein (Hcp), can cause apoptosis of the host cells through activation of caspase 3 (Suarez et al., 2008), impair granulocyte activation, and cause disseminated infections in animals (Suarez et al., 2010b). Valine-glycine-repeat G (VgrG) family proteins exert cytotoxic effects by ADP-ribosylation of actin, thus prohibiting its polymerization and cytotoxicity in host cells (Suarez et al., 2010a). Recently, the exotoxin A (ExoA) has been suggested as a marked virulence factor harbored by A. dhakensis SSU strain (Ponnusamy et al., 2016). The ExoA-associated gene also has been identified in the genome of A. dhakensis BVH28b (Mosser et al., 2015). Of note, a comparative study of whole genome sequences of six Aeromonas strains demonstrated that exo $A$ was only identified in $A$. dhakensis with particularly high virulence in C. elegans (Mosser et al., 2015). An exoA-harboring A. hydrophila strain was also found to be highly virulent to mice (Ponnusamy et al., 2016). However, the published data about this virulence factor are limited and the real prevalence of exo $A$ in $A$. dhakensis or other Aeromonas species needs to be further studied.

Aeromonas species have a variety of QSSs which could modulate bacterial virulence genes (Swift et al., 1997; Kozlova et al., 2011). Consistent with this assumption, the data from Khajanchi et al. (2009) showed that a mutation of the ahyRI 
TABLE 1 | Regulatory mechanism of putative virulent factors identified in Aeromonas dhakensis SSU strain.

\begin{tabular}{|c|c|c|}
\hline Factor & Mechanism & $\begin{array}{l}\text { Effect on } \\
\text { virulence }\end{array}$ \\
\hline Alt, heat-liable enterotoxin & $\uparrow \mathrm{cAMP}$ and prostaglandins in intestinal mucosa & $\uparrow$ \\
\hline Ast, heat-stable enterotoxin & $\uparrow c A M P$ in intestinal mucosa & $\uparrow$ \\
\hline \multicolumn{3}{|l|}{ Type 2 secretion system } \\
\hline Act, aerolysin-related cytotoxic enterotoxin & Hemolytic, cytotoxic, and enterotoxic activities & $\uparrow$ \\
\hline \multicolumn{3}{|l|}{ Type 3 secretion system } \\
\hline AexU & $\begin{array}{l}\text { GAP and ADP-ribosyltransferase, actin reorganization, apoptosis, inhibit NF-кB, inactivate Rho } \\
\text { GTPase, } \downarrow \text { secretion of IL-6 and IL-8 }\end{array}$ & $\uparrow$ \\
\hline \multicolumn{3}{|l|}{ Type 6 secretion system } \\
\hline Hcp, hemolysin coregulated protein & $\begin{array}{l}\text { Apoptosis, necrosis of cells, impair macrophage phagocytosis, } \downarrow \text { pro-inflammatory cytokines, } \\
\uparrow \text { suppressive cytokines }\end{array}$ & $\uparrow$ \\
\hline Valine-glycine-repeat G & ADP-ribosylation of actin, and cytotoxicity of cells, apoptosis & $\uparrow$ \\
\hline \multicolumn{3}{|l|}{ Quorum sensing system } \\
\hline AhyRI & $\uparrow$ Protease production and biofilm formation & $\uparrow$ \\
\hline LuxS & $\begin{array}{l}\downarrow \text { Secretion of T6SS-asscoiated effectors, } \\
\downarrow \text { protease production and biofilm formation }\end{array}$ & $\downarrow$ \\
\hline QseBC & $\begin{array}{l}\uparrow \text { Swimming and swarming motility } \\
\uparrow \text { Production of protease and cytotoxic enterotoxin } \\
\downarrow \text { Biofilm formation }\end{array}$ & $\begin{array}{l}\uparrow \\
\uparrow \\
\downarrow\end{array}$ \\
\hline
\end{tabular}

$\uparrow$, increase; $\downarrow$, decrease.

QSS of A. dhakensis SSU would impair the secretion of T6SSassociated effectors, protease production, and biofilm formation, if compared with the wild strain (Khajanchi et al., 2009). Another example concerns the biofilm formation that may help Aeromonas adhere to cell surfaces and is likely associated with gastroenteritis or wound infection (Janda and Abbott, 2010). The LuxS-based QSS inhibiting biofilm formation and decreasing virulence in a septicemic mouse model was regarded as a negative regulator of virulence in A. dhakensis SSU (Kozlova et al., 2008). Another QseBC QSS has been shown to both positively and negatively regulate various virulence factors of $A$. dhakensis SSU (Khajanchi et al., 2012; Kozlova et al., 2012). The decreased virulence of the $\triangle q s e B$ mutant of $A$. dhakensis is correlated with reduced motility, and production of proteases and cytotoxic toxin. In contrast, biofilm is overexpressed if $q s e B$ is deleted. Moreover, ahyRI, LuxS, and QseBC systems co-regulate biofilm formation and motility of $A$. dhakensis and have complicated interactions (Kozlova et al., 2015). The balance between anhyRI and QseBC systems in A. dhakensis is regulated by c-di-GMP (Kozlova et al., 2011) and c-di-GMP regulation of QseBC function relies on the expression of the ahyRI system (Kozlova et al., 2012).

Although several virulence genes were reported in the SSU strain, comparative studies on the genomes of other A. dhakensis strains (i.e., CIP 107500, AAK1, CECT 7289, and BVH28b) showed that these virulence associated genes are not always found. Despite a body of relevant evidence argues for increased virulence (Chen et al., 2014c), virulence mechanisms remain inadequately defined. Further studies based on extensive analysis of whole genome sequences of $A$. dhakensis are needed. Accurate species and virulence factors identification may offer the chances to develop novel strategies to combat severe A. dhakensis infections in humans, in addition to antibiotics.

\section{CLINICAL INFECTIONS}

Aeromonas species are often included in the list of enteropathogens and can present with invasive extra-intestinal infections, including septicemia, hepatobiliary tract infections, and soft tissue-infections. In the past, human diseases were mostly reported to be associated with three species, i.e., A. hydrophila, A. veronii, and A. caviae. However, the clinical importance given to A. hydrophila has been misguided because of the inaccurate species identification. With the introduction of molecular identification methods and the A. dhakensis description, the order of three previously predominant species has dramatically changed. A. dhakensis, accounting for $25.7 \%$ of identified isolates, was the second common species derived from the data of recent studies (Beaz-Hidalgo and Figueras, 2015). In a review of Aeromonas species distribution stratified by the isolation origin, feces was the most common origin $(40.0 \%$, $327 / 817)$, followed by wound $(29.3 \%, 239 / 817)$ and blood $(22.2 \%$, 181/817) (Beaz-Hidalgo and Figueras, 2015). Among wound isolates, A. dhakensis was the predominant species $(34.3 \%$, 82/239), followed by A. hydrophila $(27.2 \%, 65 / 239)$ and A. veronii $(16.7 \%, 40 / 239)$.

\section{Skin and Soft-Tissue Infection}

Skin and soft-tissue infections (SSTIs) caused by Aeromonas species, including cellulitis (Sanger et al., 1989; Voss et al., 1992), abscesses (Voss et al., 1992; Gold and Salit, 1993), necrotizing fasciitis (Minnaganti et al., 2000), and myonecrosis (Voss et al., 1992; Vukmir, 1992; Moses et al., 1995), are common clinical manifestations. Severe SSTIs have been reported to be mainly associated with A. hydrophila (Chao et al., 2013). However, the incidence of SSTIs caused by $A$. dhakensis may have been underestimated, as this species may be reported as A. hydrophila 
based on phenotypic tests (Lamy et al., 2010; Aravena-Roman et al., 2011; Chao et al., 2013) or prior to the species description of $A$. dhakensis. Given the recognized importance of $A$. dhakensis in diseases, epidemiologic data before the taxonomic description of $A$. dhakensis should be considered as outdated, at least for the prevalence of $A$. hydrophila. In our research, A. dhakensis was the most prevalent (46.3\%) among 80 Aeromonas wound isolates in southern Taiwan (Chen et al., 2014c). Among the 11 patients with monomicrobial Aeromonas wound infections, seven were infected by $A$. dhakensis and three evolved into necrotizing fasciitis requiring surgical fasciotomy (Chen et al., 2014c).

\section{Bacteremia}

Aeromonas bacteremia usually occurs in patients with underlying illnesses, such as malignancy, liver cirrhosis, diabetes mellitus, or receipt of immunosuppressant therapy (Tang et al., 2014). Previous studies indicated that A. hydrophila, A. caviae, and A. veronii, accounted for the majority of Aeromonas bacteremia (Chuang et al., 2011; Tang et al., 2014). Our recent work revealed that among 153 episodes of monomicrobial Aeromonas bacteremia, A. veronii (50 isolates, 32.7\%), A. dhakensis (48, $31.4 \%)$, A. caviae $(43,28.1 \%)$, and A. hydrophila (10, 6.5\%) were the principal causative species (Wu et al., 2015). The origins of $A$. dhakensis bacteremia were mainly unrecognized (47.9\%), spontaneous bacterial peritonitis (16.7\%), biliary tract infection (10.4\%), and skin and soft-tissue infection (10.4\%). A substantial proportion of $A$. dhakensis bacteremia was community-onset (70.8\%) and occurred in cirrhotic patients (62.5\%). Of note, A. dhakensis was linked to the highest 14-day sepsis-related mortality rate $(25.5 \%)$ compared with $A$. veronii $(14.0 \%)$ and A. caviae $(4.7 \%)$. Because of the distribution of $A$. dhakensis in the environment and aquatic creatures (Martinez-Murcia et al., 2008; Aravena-Roman et al., 2011), oral ingestion or abraded wounds can serve as the portals of entry of $A$. dhakensis infection.

\section{Gastroenteritis}

Aeromonas dhakensis was first isolated from children with diarrhea in Dhaka, Bangladesh (Huys et al., 2002) and the A. dhakensis SSU was recovered from a diarrheal patient during a cholera-like outbreak (Grim et al., 2014). In a review of Aeromonas species identified by molecular methods among clinical samples, $43.6 \%$ (82 of 188) of A. dhakensis were isolated from feces (Beaz-Hidalgo and Figueras, 2015). In Taiwan, $\mathrm{Wu}$ et al. (2012b) reported a cirrhotic patient with bloody diarrhea, in whom $A$. dhakensis identified using $r p o D$ and $r p o B$ sequencing was concurrently isolated from blood and feces. Significant $A$. dhakensis-induced cytotoxicity to intestinal epithelial cells was demonstrated in vitro. Among 11 Taiwanese adults with Aeromonas-associated diarrhea, only one A. dhakensis isolate was obtained from an 83-year-old female with an uneventful hospital course (Chen et al., 2014b). Most of the fecal isolates of $A$. dhakensis originated from Asia or tropical areas, suggestive of geographic variation of prevalence.

\section{ANTIMICROBIAL RESISTANCE AND THERAPY}

Aeromonas dhakensis is susceptible to cefepime, aminoglycosides, fluoroquinolones, and tetracyclines (Chen et al., 2014c; Wu et al., 2015). Although antimicrobial resistance of broadspectrum cephalosporins or carbapenems was not common in A. dhakensis isolates (Chen et al., 2014a,c; Wu et al., 2015), this species intrinsically harbors class B (metallo- $\beta$-lactamases, MBLs; CphA), C (AmpC cephalosporinase; AQU-1), and D $\beta$-lactamases (penicillinases; Wu et al., 2012a, 2013). CphA, a MBL found in A. dhakensis, A. veronii, and A. hydrophila (Wu et al., 2012a), has a specific substrate profile of hydrolyzing carbapenems but not penicillins or cephalosporins, if compared with other MBLs (Segatore et al., 1993). However, CphA carbapenemase production is not easily detected by the conventional susceptibility test, unless a large inoculum or additional tests (e.g., modified Hodge test, ertapenem MIC or Carba NP test) are applied (Rossolini et al., 1995; Wu et al., 2012a; Sinclair et al., 2016). Carbapenem therapy for cphAcarrying Aeromonas infections remains controversial, because of the clinical concern that carbapenem monotherapy may fail in treating MBL-producing aeromonads infections with a high bacterial burden, such as peritonitis/abdominal sepsis or soft tissue infections (Wu et al., 2012a). Moreover, breakthrough bacteremia due to $A$. dhakensis during ertapenem therapy has been reported (Wu et al., 2012a). Therefore, performing the susceptibility tests with a large inoculum and/or with additional tests before carbapenem therapy is advised for severe A. dhakensis infections. Optimal antimicrobial choices for A. dhakensis infections may include a fluoroquinolone or cefepime before the drug susceptibility is conclusive.

AmpC $\beta$-lactamases can hydrolyze cephamycins and third generation cephalosporins, and are resistant to $\beta$-lactamase inhibitors, such as clavulanic acid, tazobactam, and sulbactam (Bush et al., 1995). The distribution of the AmpC $\beta$-lactamase among Aeromonas strains is species-specific, i.e., bla $a_{\mathrm{AQU}-1}$, $b l a_{\mathrm{MOX}}$, or bla $a_{\mathrm{CepH}}$ was present in A. dhakensis, A. caviae, or A. hydrophila, respectively (Wu et al., 2015). These findings suggest that clinical use of broad-spectrum cephalosporins, except cefepime, for $A$. dhakensis infections, should be used with caution before supporting antimicrobial susceptibility testing data are available.

\section{CONCLUSION}

Aeromonas dhakensis is a demonstrative example that accurate taxonomy can improve our knowledge about epidemiological distribution and virulence potential of human pathogens. At present time, accurate identification of $A$. dhakensis is encouraged because of its virulence and potential antimicrobial resistance. Consequently, improved recognition of $A$. dhakensis will lead to revise our considerations on actual pathogenic potential and clinical impact of $A$. hydrophila infection. More researches to re-estimate clinical role of $A$. hydrophila in human infection, to disclose disease burden of $A$. dhakensis, and to understand how 
this enhanced virulent species could emerge from the Aeromonas complex, are warranted.

\section{AUTHOR CONTRIBUTIONS}

Composed the article: PLC; drafted the paper: PLC; critically commented and revised the manuscript: WCK and BL. All authors read and approved the final manuscript.

\section{REFERENCES}

Aravena-Roman, M., Harnett, G. B., Riley, T. V., Inglis, T. J., and Chang, B. J. (2011). Aeromonas aquariorum is widely distributed in clinical and environmental specimens and can be misidentified as Aeromonas hydrophila. J. Clin. Microbiol. 49, 3006-3008. doi: 10.1128/JCM.00472-11

Beaz-Hidalgo, R., and Figueras, M. J. (2015). “Aeromonas infections in humans," in Aeromonas, ed. J. Graf (Portland, OR: Caister Academic Press), 65-108.

Beaz-Hidalgo, R., Hossain, M. J., Liles, M. R., and Figueras, M. J. (2015). Strategies to avoid wrongly labelled genomes using as example the detected wrong taxonomic affiliation for aeromonas genomes in the GenBank database. PLoS ONE 10:e0115813. doi: 10.1371/journal.pone.0115813

Beaz-Hidalgo, R., Martinez-Murcia, A., and Figueras, M. J. (2013). Reclassification of Aeromonas hydrophila subsp. dhakensis Huys et al., 2002 and Aeromonas aquariorum Martinez-Murcia et al., 2008 as Aeromonas dhakensis sp. nov. comb nov. and emendation of the species Aeromonas hydrophila. Syst. Appl. Microbiol. 36, 171-176. doi: 10.1016/j.syapm.2012.12.007

Bush, K., Jacoby, G. A., and Medeiros, A. A. (1995). A functional classification scheme for beta-lactamases and its correlation with molecular structure. Antimicrob. Agents Chemother. 39, 1211-1233. doi: 10.1128/AAC.39.6.1211

Chao, C. M., Lai, C. C., Gau, S. J., and Hsueh, P. R. (2013). Skin and soft tissue infection caused by Aeromonas species in cancer patients. J. Microbiol. Immunol. Infect. 46, 144-146. doi: 10.1016/j.jmii.2013.02.006

Chen, P. L., Lee, T. F., Wu, C. J., Teng, S. H., Teng, L. J., Ko, W. C., et al. (2014a). Matrix-assisted laser desorption ionization-time of flight mass spectrometry can accurately differentiate Aeromonas dhakensis from A. hydrophila, A. caviae, and A. veronii. J. Clin. Microbiol. 52, 2625-2628. doi: 10.1128/JCM.01025-14

Chen, P. L., Tsai, P. J., Chen, C. S., Lu, Y. C., Chen, H. M., Lee, N. Y., et al. (2014b). Aeromonas stool isolates from individuals with or without diarrhea in southern Taiwan: predominance of Aeromonas veronii. J. Microbiol. Immunol. Infect. 48, 618-624. doi: 10.1016/j.jmii.2014.08.007

Chen, P. L., Wu, C. J., Chen, C. S., Tsai, P. J., Tang, H. J., and Ko, W. C. (2014c). A comparative study of clinical Aeromonas dhakensis and Aeromonas hydrophila isolates in southern Taiwan: A. dhakensis is more predominant and virulent. Clin. Microbiol. Infect. 20, O428-O434. doi: 10.1111/1469-0691. 12456

Chen, P. L., Wu, C. J., Tsai, P. J., Tang, H. J., Chuang, Y. C., Lee, N. Y., et al. (2014d). Virulence diversity among bacteremic Aeromonas isolates: ex vivo, animal, and clinical evidences. PLoS ONE 9:e111213. doi: 10.1371/journal.pone.0111213

Chopra, A. K., and Houston, C. W. (1999). Enterotoxins in Aeromonasassociated gastroenteritis. Microbes Infect. 1, 1129-1137. doi: 10.1016/S12864579(99)00202-6

Chuang, H. C., Ho, Y. H., Lay, C. J., Wang, L. S., Tsai, Y. S., and Tsai, C. C. (2011). Different clinical characteristics among Aeromonas hydrophila, Aeromonas veronii biovar sobria and Aeromonas caviae monomicrobial bacteremia. J. Korean Med. Sci. 26, 1415-1420. doi: 10.3346/jkms.2011.26.11.1415

Colston, S. M., Fullmer, M. S., Beka, L., Lamy, B., Gogarten, J. P., and Graf, J. (2014). Bioinformatic genome comparisons for taxonomic and phylogenetic assignments using Aeromonas as a test case. MBio 5:e02136. doi: 10.1128/mBio.02136-14

Donohue, M. J., Best, J. M., Smallwood, A. W., Kostich, M., Rodgers, M., and Shoemaker, J. A. (2007). Differentiation of Aeromonas isolated from drinking water distribution systems using matrix-assisted laser desorption/ionizationmass spectrometry. Anal. Chem. 79, 1939-1946. doi: 10.1021/ac0611420

Esteve, C., Alcaide, E., and Blasco, M. D. (2012). Aeromonas hydrophila subsp. dhakensis isolated from feces, water and fish in Mediterranean Spain. Microbes Environ. 27, 367-373. doi: 10.1264/jsme2.ME12009

\section{ACKNOWLEDGMENT}

The study was partially supported by research grants from the Ministry of Science and Technology (MOST 1032314-B-006-076-MY2), Ministry of Health and Welfare (MOHW105-TDU-B-211-113002), and National Cheng Kung University Hospital (NCKUH-10408015 and NCKUH10507004).

Figueras, M. J., Alperi, A., Saavedra, M. J., Ko, W. C., Gonzalo, N., Navarro, M., et al. (2009). Clinical relevance of the recently described species Aeromonas aquariorum. J. Clin. Microbiol. 47, 3742-3746. doi: 10.1128/JCM.02216-08

Figueras, M. J., Beaz-Hidalgo, R., Senderovich, Y., Laviad, S., and Halpern, M. (2011). Re-identification of Aeromonas isolates from chironomid egg masses as the potential pathogenic bacteria Aeromonas aquariorum. Environ. Microbiol. Rep. 3, 239-244. doi: 10.1111/j.1758-2229.2010.00216.x

Gold, W. L., and Salit, I. E. (1993). Aeromonas hydrophila infections of skin and soft tissue: report of 11 cases and review. Clin. Infect. Dis. 16, 69-74. doi: 10.1093/clinids/16.1.69

Grim, C. J., Kozlova, E. V., Ponnusamy, D., Fitts, E. C., Sha, J., Kirtley, M. L., et al. (2014). Functional Genomic characterization of virulence factors from necrotizing fasciitis-causing strains of Aeromonas hydrophila. Appl. Environ. Microbiol. 80, 4162-4183. doi: 10.1128/AEM.00486-14

Huys, G., Kampfer, P., Albert, M. J., Kuhn, I., Denys, R., and Swings, J. (2002). Aeromonas hydrophila subsp. dhakensis subsp. nov., isolated from children with diarrhoea in Bangladesh, and extended description of Aeromonas hydrophila subsp. hydrophila (Chester 1901) Stanier 1943 (approved lists 1980). Int. J. Syst. Evol. Microbiol. 52, 705-712. doi: 10.1099/ijs.0.01844-0

Huys, G., Pearson, M., Kampfer, P., Denys, R., Cnockaert, M., Inglis, V., et al. (2003). Aeromonas hydrophila subsp. ranae subsp. nov., isolated from septicaemic farmed frogs in Thailand. Int. J. Syst. Evol. Microbiol. 53, 885-891. doi: 10.1099/ijs.0.02357-0

Jagoda, S. S., Wijewardana, T. G., Arulkanthan, A., Igarashi, Y., Tan, E., Kinoshita, S., et al. (2014). Characterization and antimicrobial susceptibility of motile aeromonads isolated from freshwater ornamental fish showing signs of septicaemia. Dis. Aquat. Organ. 109, 127-137. doi: 10.3354/dao 02733

Janda, J. M., and Abbott, S. L. (2007). 16S rRNA gene sequencing for bacterial identification in the diagnostic laboratory: pluses, perils, and pitfalls. J. Clin. Microbiol. 45, 2761-2764. doi: 10.1128/JCM.01228-07

Janda, J. M., and Abbott, S. L. (2010). The genus Aeromonas: taxonomy, pathogenicity, and infection. Clin. Microbiol. Rev. 23, 35-73. doi: 10.1128/CMR.00039-09

Khajanchi, B. K., Kozlova, E. V., Sha, J., Popov, V. L., and Chopra, A. K. (2012). The two-component QseBC signalling system regulates in vitro and in vivo virulence of Aeromonas hydrophila. Microbiology 158, 259-271. doi: 10.1099/mic.0.051805-0

Khajanchi, B. K., Sha, J., Kozlova, E. V., Erova, T. E., Suarez, G., Sierra, J. C., et al. (2009). N-acylhomoserine lactones involved in quorum sensing control the type VI secretion system, biofilm formation, protease production, and in vivo virulence in a clinical isolate of Aeromonas hydrophila. Microbiology 155, 3518-3531. doi: 10.1099/mic.0.031575-0

Kozlova, E. V., Khajanchi, B. K., Popov, V. L., Wen, J., and Chopra, A. K. (2012). Impact of QseBC system in c-di-GMP-dependent quorum sensing regulatory network in a clinical isolate SSU of Aeromonas hydrophila. Microb. Pathog. 53, 115-124. doi: 10.1016/j.micpath.2012.05.008

Kozlova, E. V., Khajanchi, B. K., Sha, J., and Chopra, A. K. (2011). Quorum sensing and c-di-GMP-dependent alterations in gene transcripts and virulenceassociated phenotypes in a clinical isolate of Aeromonas hydrophila. Microb. Pathog. 50, 213-223. doi: 10.1016/j.micpath.2011.01.007

Kozlova, E. V., Ponnusamy, D., and Chopra, A. K. (2015). "New developments on the virulence mechanisms of Aeromonas hydrophila," in Aeromonas, ed. J. Graf (Norfolk: Caister Academic Press), 139-153.

Kozlova, E. V., Popov, V. L., Sha, J., Foltz, S. M., Erova, T. E., Agar, S. L., et al. (2008). Mutation in the S-ribosylhomocysteinase (luxS) gene involved in quorum sensing affects biofilm formation and virulence in a clinical isolate of Aeromonas 
hydrophila. Microb. Pathog. 45, 343-354. doi: 10.1016/j.micpath.2008. 08.007

Lamy, B., Kodjo, A., and Laurent, F. (2011). Identification of Aeromonas isolates by matrix-assisted laser desorption ionization time-of-flight mass spectrometry. Diagn. Microbiol. Infect. Dis. 71, 1-5. doi: 10.1016/j.diagmicrobio.2011. 04.014

Lamy, B., Laurent, F., and Kodjo, A. (2010). Validation of a partial rpoB gene sequence as a tool for phylogenetic identification of aeromonads isolated from environmental sources. Can. J. Microbiol. 56, 217-228. doi: 10.1139/w $10-006$

Martinez-Murcia, A., Monera, A., Alperi, A., Figueras, M. J., and Saavedra, M. J. (2009). Phylogenetic evidence suggests that strains of Aeromonas hydrophila subsp. dhakensis belong to the species Aeromonas aquariorum sp. nov. Curr. Microbiol. 58, 76-80. doi: 10.1007/s00284-008-9278-6

Martinez-Murcia, A. J., Benlloch, S., and Collins, M. D. (1992). Phylogenetic interrelationships of members of the genera Aeromonas and Plesiomonas as determined by $16 \mathrm{~S}$ ribosomal DNA sequencing: lack of congruence with results of DNA-DNA hybridizations. Int. J. Syst. Bacteriol. 42, 412-421. doi: 10.1099/00207713-42-3-412

Martinez-Murcia, A. J., Monera, A., Saavedra, M. J., Oncina, R., Lopez-Alvarez, M., Lara, E., et al. (2011). Multilocus phylogenetic analysis of the genus Aeromonas. Syst. Appl. Microbiol. 34, 189-199. doi: 10.1016/j.syapm.2010.11.014

Martinez-Murcia, A. J., Saavedra, M. J., Mota, V. R., Maier, T., Stackebrandt, E., and Cousin, S. (2008). Aeromonas aquariorum sp. nov., isolated from aquaria of ornamental fish. Int. J. Syst. Evol. Microbiol. 58, 1169-1175. doi: 10.1099/ijs.0.65352-0

Martino, M. E., Fasolato, L., Montemurro, F., Rosteghin, M., Manfrin, A., Patarnello, T., et al. (2011). Determination of microbial diversity of Aeromonas strains on the basis of multilocus sequence typing, phenotype, and presence of putative virulence genes. Appl. Environ. Microbiol. 77, 4986-5000. doi: 10.1128/AEM.00708-11

Minnaganti, V. R., Patel, P. J., Iancu, D., Schoch, P. E., and Cunha, B. A. (2000). Necrotizing fasciitis caused by Aeromonas hydrophila. Heart Lung 29, 306-308. doi: $10.1067 / \mathrm{mhl} .2000 .106723$

Morinaga, Y., Yanagihara, K., Araki, N., Harada, Y., Yamada, K., Akamatsu, N., et al. (2011a). Clinical characteristics of seven patients with Aeromonas septicemia in a Japanese hospital. Tohoku J. Exp. Med. 225, 81-84. doi: $10.1620 /$ tjem.225.81

Morinaga, Y., Yanagihara, K., Eugenin, F. L., Beaz-Hidalgo, R., Kohno, S., and Figueras Salvat, M. J. (2011b). Identification error of Aeromonas aquariorum: a causative agent of septicemia. Diagn. Microbiol. Infect. Dis. 76, 106-109. doi: 10.1016/j.diagmicrobio.2013.01.019

Moses, A. E., Leibergal, M., Rahav, G., Perouansky, M., Or, R., and Shapiro, M. (1995). Aeromonas hydrophila myonecrosis accompanying mucormycosis five years after bone marrow transplantation. Eur. J. Clin. Microbiol. Infect. Dis. 14, 237-240. doi: 10.1007/BF02310364

Mosser, T., Talagrand-Reboul, E., Colston, S. M., Graf, J., Figueras, M. J., JumasBilak, E., et al. (2015). Exposure to pairs of Aeromonas strains enhances virulence in the Caenorhabditis elegans infection model. Front. Microbiol. 6:1218. doi: 10.3389/fmicb.2015.01218

Perez, L., Abarca, M. L., Latif-Eugenin, F., Beaz-Hidalgo, R., Figueras, M. J., and Domingo, M. (2015). Aeromonas dhakensis pneumonia and sepsis in a neonate Risso's dolphin Grampus griseus from the Mediterranean Sea. Dis. Aquat. Organ. 116, 69-74. doi: 10.3354/dao02899

Ponnusamy, D., Kozlova, E. V., Sha, J., Erova, T. E., Azar, S. R., Fitts, E. C., et al. (2016). Cross-talk among flesh-eating Aeromonas hydrophila strains in mixed infection leading to necrotizing fasciitis. Proc. Natl. Acad. Sci. U.S.A. 113, 722-727. doi: 10.1073/pnas.1523817113

Puthucheary, S. D., Puah, S. M., and Chua, K. H. (2012). Molecular characterization of clinical isolates of Aeromonas species from Malaysia. PLoS ONE 7:e30205. doi: 10.1371/journal.pone.0030205

Roger, F., Lamy, B., Jumas-Bilak, E., Kodjo, A., Laurent, F., colBVH study group, et al. (2012a). Ribosomal multi-operon diversity: an original perspective on the genus Aeromonas. PLoS ONE 7:e46268. doi: 10.1371/journal.pone. 0046268

Roger, F., Marchandin, H., Jumas-Bilak, E., Kodjo, A., the colBVH study group, and Lamy, B. (2012b). Multilocus genetics to reconstruct aeromonad evolution. BMC Microbiol. 12:62. doi: 10.1186/1471-2180-12-62
Rossolini, G. M., Zanchi, A., Chiesurin, A., Amicosante, G., Satta, G., and Guglielmetti, P. (1995). Distribution of cphA or related carbapenemaseencoding genes and production of carbapenemase activity in members of the genus Aeromonas. Antimicrob. Agents Chemother. 39, 346-349. doi: 10.1128/AAC.39.2.346

Sanger, J. R., Yousif, N. J., and Matloub, H. S. (1989). Aeromonas hydrophila upper extremity infection. J. Hand Surg. Am. 14, 719-721. doi: 10.1016/03635023(89)90198-6

Sedlacek, I., Krejci, E., Andelova, A., Sedlackova, M., Porazilova, I., and Holochova, P. (2012). Aeromonas hydrophila subsp. dhakensis-a causative agent of gastroenteritis imported into the Czech Republic. Ann. Agric. Environ. Med. 19, 409-413.

Segatore, B., Massidda, O., Satta, G., Setacci, D., and Amicosante, G. (1993). High specificity of cphA-encoded metallo-beta-lactamase from Aeromonas hydrophila AE036 for carbapenems and its contribution to beta-lactam resistance. Antimicrob. Agents Chemother. 37, 1324-1348. doi: 10.1128/AAC.37.6.1324

Sha, J., Kozlova, E. V., and Chopra, A. K. (2002). Role of various enterotoxins in Aeromonas hydrophila-induced gastroenteritis: generation of enterotoxin genedeficient mutants and evaluation of their enterotoxic activity. Infect. Immun. 70, 1924-1935. doi: 10.1128/IAI.70.4.1924-1935.2002

Sha, J., Wang, S. F., Suarez, G., Sierra, J. C., Fadl, A. A., Erova, T. E., et al. (2007). Further characterization of a type III secretion system (T3SS) and of a new effector protein from a clinical isolate of Aeromonas hydrophila-part I. Microb. Pathog. 43, 127-146. doi: 10.1016/j.micpath.2007.05.002

Shin, G. W., You, M. J., Cho, H. S., Yi, S. W., and Lee, C. S. (2013). Severe sepsis due to Aeromonas aquariorum in a patient with liver cirrhosis. Jpn. J. Infect. Dis. 66, 519-522. doi: 10.7883/yoken.66.519

Sinclair, H. A., Heney, C., Sidjabat, H. E., George, N. M., Bergh, H., Anuj, S. N., et al. (2016). Genotypic and phenotypic identification of Aeromonas species and CphA-mediated carbapenem resistance in Queensland, Australia. Diagn. Microbiol. Infect. Dis. doi: 10.1016/j.diagmicrobio.2016.02.005 [Epub ahead of print].

Soto-Rodriguez, S. A., Cabanillas-Ramos, J., Alcaraz, U., Gomez-Gil, B., and Romalde, J. L. (2011). Identification and virulence of Aeromonas dhakensis, Pseudomonas mosselii and Microbacterium paraoxydans isolated from Nile tilapia, Oreochromis niloticus, cultivated in Mexico. J. Appl. Microbiol. 115, 654-662. doi: 10.1111/jam.12280

Suarez, G., Sierra, J. C., Erova, T. E., Sha, J., Horneman, A. J., and Chopra, A. K. (2010a). A type VI secretion system effector protein, VgrG1, from Aeromonas hydrophila that induces host cell toxicity by ADP ribosylation of actin. J. Bacteriol. 192, 155-168. doi: 10.1128/JB.01260-09

Suarez, G., Sierra, J. C., Kirtley, M. L., and Chopra, A. K. (2010b). Role of Hcp, a type 6 secretion system effector, of Aeromonas hydrophila in modulating activation of host immune cells. Microbiology 156, 3678-3688. doi: 10.1099/mic.0.041277-0

Suarez, G., Sierra, J. C., Sha, J., Wang, S., Erova, T. E., Fadl, A. A., et al. (2008). Molecular characterization of a functional type VI secretion system from a clinical isolate of Aeromonas hydrophila. Microb. Pathog. 44, 344-361. doi: 10.1016/j.micpath.2007.10.005

Swift, S., Karlyshev, A. V., Fish, L., Durant, E. L., Winson, M. K., Chhabra, S. R., et al. (1997). Quorum sensing in Aeromonas hydrophila and Aeromonas salmonicida: identification of the LuxRI homologs AhyRI and AsaRI and their cognate $\mathrm{N}$-acylhomoserine lactone signal molecules. J. Bacteriol. 179, 5271-5281.

Tang, H. J., Lai, C. C., Lin, H. L., and Chao, C. M. (2014). Clinical manifestations of bacteremia caused by Aeromonas species in southern Taiwan. PLoS ONE 9:e91642. doi: 10.1371/journal.pone.0091642

Tomas, J. M. (2012). The main Aeromonas pathogenic factors. ISRN Microbiol. 2012, 256261. doi: $10.5402 / 2012 / 256261$

Voss, L. M., Rhodes, K. H., and Johnson, K. A. (1992). Musculoskeletal and soft tissue Aeromonas infection: an environmental disease. Mayo Clin. Proc. 67, 422-427. doi: 10.1016/S0025-6196(12)60387-5

Vukmir, R. B. (1992). Aeromonas hydrophila: myofascial necrosis and sepsis. Intensive Care Med. 18, 172-174. doi: 10.1007/BF01709242

Wu, C. J., Chen, P. L., Hsueh, P. R., Chang, M. C., Tsai, P. J., Shih, H. I., et al. (2015). Clinical implications of species identification in monomicrobial Aeromonas bacteremia. PLoS ONE 10:e0117821. doi: 10.1371/journal.pone.0117821 
Wu, C. J., Chen, P. L., Wu, J. J., Yan, J. J., Lee, C. C., Lee, H. C., et al. (2012a). Distribution and phenotypic and genotypic detection of a metallo-betalactamase, CphA, among bacteraemic Aeromonas isolates. J. Med. Microbiol. 61, 712-719. doi: 10.1099/jmm.0.038323-0

Wu, C. J., Tsai, P. J., Chen, P. L., Wu, I. C., Lin, Y. T., Chen, Y. H., et al. (2012b). Aeromonas aquariorum septicemia and enterocolitis in a cirrhotic patient. Diagn. Microbiol. Infect. Dis. 74, 406-408. doi: 10.1016/j.diagmicrobio.2012.08.005

Wu, C. J., Wang, H. C., Chen, P. L., Chang, M. C., Sunny Sun, H., Chou, P. H., et al. (2013). AQU-1, a chromosomal class C beta-lactamase, among clinical Aeromonas dhakensis isolates: distribution and clinical significance. Int. J. Antimicrob. Agents 42, 456-461. doi: 10.1016/j.ijantimicag.2013.08.002

Xu, X. J., Ferguson, M. R., Popov, V. L., Houston, C. W., Peterson, J. W., and Chopra, A. K. (1998). Role of a cytotoxic enterotoxin in Aeromonas-mediated infections: development of transposon and isogenic mutants. Infect. Immun. 66, 3501-3509.

Yano, Y., Hamano, K., Tsutsui, I., Aue-Umneoy, D., Ban, M., and Satomi, M. (2015). Occurrence, molecular characterization, and antimicrobial susceptibility of Aeromonas spp. in marine species of shrimps cultured at inland low salinity ponds. Food Microbiol. 47, 21-27. doi: 10.1016/j.fm.2014.11.003

Yi, S. W., You, M. J., Cho, H. S., Lee, C. S., Kwon, J. K., and Shin, G. W. (2013). Molecular characterization of Aeromonas species isolated from farmed eels (Anguilla japonica). Vet. Microbiol. 164, 195-200. doi: 10.1016/j.vetmic.2013.02.006

Conflict of Interest Statement: The authors declare that the research was conducted in the absence of any commercial or financial relationships that could be construed as a potential conflict of interest.

Copyright (0) 2016 Chen, Lamy and Ko. This is an open-access article distributed under the terms of the Creative Commons Attribution License (CC BY). The use, distribution or reproduction in other forums is permitted, provided the original author(s) or licensor are credited and that the original publication in this journal is cited, in accordance with accepted academic practice. No use, distribution or reproduction is permitted which does not comply with these terms. 